\title{
Spray cryotherapy for dysphagia palliation in esophageal cancer prior to systemic therapy: is it ready for prime time?
}

\section{다)(요 $\odot$}

\author{
Author \\ Bruce D. Greenwald \\ Institution \\ University of Maryland School of Medicine and Marlene and \\ Stewart Greenebaum Comprehensive Cancer Center, \\ Baltimore, Maryland, United States \\ Bibliography \\ DOI https://doi.org/10.1055/a-0966-8457 | \\ Endoscopy International Open 2020; 08: E122-E123 \\ (c) Georg Thieme Verlag KG Stuttgart · New York \\ eISSN 2196-9736
}

\author{
Corresponding author \\ Bruce D. Greenwald, MD, Professor of Medicine, University \\ of Maryland School of Medicine and Marlene and Stewart \\ Greenebaum Comprehensive Cancer Center, 22 S. Greene \\ Street, N3W62, Baltimore, MD 21201-1595 \\ Fax: +1-410-328-8315 \\ bgreenwa@som.umaryland.edu
}

Dysphagia is a common presenting symptom in esophageal cancer. Poor nutrition and diminished quality of life are often the result. Traditional options to manage dysphagia - esophageal stents, radiation, chemotherapy, and dilation - all have limitations. Liquid nitrogen spray cryotherapy (truFreeze, CSA Medical, Inc., Lexington, Massachusetts, United States) has been used successfully to treat inoperable early-stage esophageal cancer and to palliate dysphagia in locally advanced tumors [1]. This non-contact method uses liquid nitrogen at $-196^{\circ}$ to effectively freeze and destroy esophageal tumors with excellent tolerability and safety profile.

In a recent multicenter retrospective study, endoscopic liquid nitrogen spray cryotherapy was used to palliate dysphagia in 49 patients with inoperable (mostly stage IV) esophageal cancer [2]. A significant improvement in dysphagia score was reported in the majority of patients. Prior to cryotherapy, median dysphagia score was 2.6 ( 1 = dysphagia to solids, 2 = dysphagia to semi-solids, 3 =dysphagia to liquids), while after treatment, this score significantly improved to 1.7. Forty-one percent of those treated had a dysphagia score $\leq 1$ after their last treatment. Cryotherapy may also induce tumor necrosis through immunologic mechanisms [3]. Cryotherapy-induced cancer cell lysis releases intact intracellular contents, which may induce a specific antitumor immune response. While this response has not been studied specifically in esophageal cancer, it may serve as an additional mechanism of antitumor therapy in these patients.

Concurrent systemic chemotherapy and external beam radiotherapy (chemoradiation) with or without esophagectomy is the primary therapy offered for non-metastatic advanced stage esophageal cancer. However, complete response to ther- apy is seen in fewer than $50 \%$ of patients in multiple studies. Attempts to improve response with increased radiation dose or alternative chemotherapy regimens have not been successful. Dysphagia palliation does occur with chemoradiation, but it typically takes several weeks before relief of dysphagia is reported. In those with severe dysphagia, chemoradiation may be delayed due to malnutrition and poor performance status. Options to improve dysphagia and nutrition in this setting include self-expanding metal stents or enteral feeding tubes (gastrostomy or jejunostomy). Feeding tubes provide no relief of dysphagia but can improve nutritional status. Esophageal stents, while often providing rapid dysphagia relief, are associated with pain, gastroesophageal reflux (for distal tumors), stent migration, and perhaps worse outcomes [4].

In this issue, Shah et al evaluate the safety and efficacy of a single treatment with endoscopic liquid nitrogen spray cryotherapy for improvement in dysphagia in 25 patients with treatment-naïve esophageal cancer [5]. In some cases, treatment was performed at the time of staging by endoscopic ultrasound (EUS), while in others it was administered in a separate session. Spray cryotherapy was used as primary treatment for dysphagia or as a "bridge" treatment to improve swallowing until systemic therapy was given. As expected, the treatment was safe, with no cryotherapy-related serious adverse events. Improvement in dysphagia was seen in $71 \%$ at 1 week and $50 \%$ in 2 weeks, with improvement at 4 weeks persisting in nearly half of the patients. Chemoradiation was administered in nine patients at a median time of 27 days after cryotherapy. None of these patients required a stent, but two required a jejunostomy tube at 4 weeks due to return of dysphagia prior to chemoradiation. Following chemoradiation, the authors note that $67 \%$ of pa- 
tients had a complete local response (no tumor cells on biopsy) and $50 \%$ had a clinical response (no tumor cells on biopsy and no evidence of nodal or metastatic disease), as assessed by endoscopy with biopsy and positron emission tomography (PET) scan, and they postulate that an immune mechanism may have contributed to these high response rates. These results must be interpreted with caution due to small numbers of patients, low negative predictive value of endoscopy and PET to predict residual persistent tumor, and absence of esophagectomy data to confirm pathologic response.

Based on these data, liquid nitrogen spray cryotherapy appears to be a reasonable alternative to esophageal stenting for patients with treatment-naïve esophageal cancer who require palliation of dysphagia, including prior to initiation of systemic therapy. The treatment is safe and can be administered at the time of staging EUS. Persistent improvement in swallowing was seen in many patients, providing relief until the effects of systemic therapy can occur. Earlier studies have shown that intermittent cryotherapy can provide prolonged relief of dysphagia in those not suitable for systemic therapy. Compared to stenting, pain and reflux are less common, although the degree of dysphagia relief may not be as great. At the current time, spray cryotherapy is only available in the United States, so widespread use is limited. Larger studies will be helpful to confirm the beneficial effect of cryotherapy as primary therapy for dysphagia and as an adjuvant to systemic therapy. Further investi- gation into potential immune-mediated cell death are warranted as well, especially now that checkpoint inhibitors and other immunotherapies are becoming widely available.

\section{Competing interests}

Dr. Greenwald is a consultant for and receives research funding from CSA Medical, Inc.

\section{References}

[1] Tsai FC, Ghorbani S, Greenwald BD et al. Safety and efficacy of endoscopic spray cryotherapy for esophageal cancer. Dis Esoph 2017; 30: 1

[2] Kachaamy T, Prakash R, Kundranda M et al. Liquid nitrogen spray cryotherapy for dysphagia palliation in patients with inoperable esophageal cancer. Gastrointest Endosc 2018; 88: 447

[3] Aarts BM, Klompenhouwer EG, Rice SL et al. Cryoablation and immunotherapy: an overview of evidence on its synergy. Insights Imaging 2019; 10: 53

[4] Dai Y, Li C, Xie Y et al. Interventions for dysphagia in oesophageal cancer. Cochrane Database Syst Rev 2014; 10: CD005048

[5] Shah T, Kushnir V, Mutha P etal. Neoadjuvant cryotherapy improves dysphagia and may impact remission rates in advanced esophageal cancer. Endosc Int Open 2019; 07: E1522 -E1527 